Jolanta Jałocha, Department of Mathematics, pl. Weyssenhoffa 11, 85-072

Bydgoszcz, Poland.

\title{
QUOTIENTS OF DARBOUX FUNCTIONS
}

\begin{abstract}
We prove theorems concerning common divisor for the families of the quotients of Darboux functions with respect to Darboux property.
\end{abstract}

\section{Introduction}

The letter $\mathbb{R}$ denotes the real line. The family of all functions from a set $X$ into $Y$ is denoted by $Y^{X}$. The word function denotes a mapping from $\mathbb{R}$ to $\mathbb{R}$ unless otherwise explicitly stated. We consider cardinals as ordinals not in one-toone correspondence with the smaller ordinals. The symbol card $X$ stands for the cardinality of a set $X$. We write $\mathfrak{c}=\operatorname{card} \mathbb{R}$. For a cardinal number $\kappa$ we write $\operatorname{cf}(\kappa)$ for the cofinality of $\kappa$, and we say that $\kappa$ is regular, if $\kappa=\operatorname{cf}(\kappa)$. The projection of a set $U \subset \mathbb{R}^{2}$ onto the $x$-axis is denoted by $\operatorname{dom} U$. We say that a set $A \subset \mathbb{R}$ is bilaterally $\mathfrak{c}$-dense in itself if $\operatorname{card}(A \cap I)=\mathfrak{c}$ for every nondegenerate interval $I$ with $A \cap I \neq \emptyset$.

Let $f: \mathbb{R} \rightarrow \mathbb{R}$. For each $y \in \mathbb{R}$ let $[f<y]=\{x \in \mathbb{R}: f(x)<y\}$. Similarly we define the symbols $[f>y],[f=y]$, etc.

The symbol $\mathcal{D}$ denotes the class of all Darboux functions; i.e., $f \in \mathcal{D}$ iff it has the intermediate value property.

There are several papers concerning theorems on a common summand [2], [1], or factor [6]. In this paper we are concerned with a common divisor for the families of the quotients of Darboux functions with respect to the Darboux property. (We were concerned with a similar problem in [3].) More precisely, we examine the cardinal

$$
\mathrm{q}(\mathcal{D}) \stackrel{\mathrm{df}}{=} \min \left\{\operatorname{card} \mathcal{F}: \mathcal{F} \subset \mathcal{D} / \mathcal{D} \& \neg\left(\exists_{g} \forall_{f \in \mathcal{F}} f / g \in \mathcal{D}\right)\right\},
$$

where

$$
\mathcal{D} / \mathcal{D} \stackrel{\text { df }}{=}\{f / g: f, g \in \mathcal{D} \& g(x) \neq 0 \text { for each } x \in \mathbb{R}\} .
$$

Key Words: Darboux function, quotient of functions.

Mathematical Reviews subject classification: Primary 26A15. Secondary 54C08.

Received by the editors June 6,2000 
In the above definition it is quite natural to restrict ourselves to subfamilies of $\mathcal{D} / \mathcal{D}$ only. Indeed, if there is a function $g$ such that both $f / g$ and $1 / g$ are Darboux, then $f \in \mathcal{D} / \mathcal{D}$.

\section{Main Results}

Before we start our examination, recall the following theorem proved by Natkaniec and Orwat [7, Theorem 7].

Theorem 2.1. Let $f$ be a function. Then $f \in \mathcal{D} / \mathcal{D}$ iff $f$ satisfies the following conditions:

D1. if $a<b$ and $f(a) f(b)<0$, then $[f=0] \cap(a, b) \neq \emptyset$;

D2. each of the sets $[f>0]$ and $[f<0]$ is bilaterally $\mathfrak{c}$-dense in itself.

Theorem 2.2. $\mathrm{q}(\mathcal{D})>\mathfrak{c}$.

Proof. Let $\left\{f_{\alpha}: \alpha<\mathfrak{c}\right\} \subset \mathcal{D} / \mathcal{D}$. For $\alpha<\mathfrak{c}$ and $i<2$ define

$$
Q_{\alpha i}=\left\{[a, b] \cap\left[(-1)^{i} f_{\alpha}>0\right]: a, b \in \mathbb{R}\right\} \backslash\{\emptyset\},
$$

and observe that by $\mathrm{D} 2$, $\operatorname{card} A=\mathfrak{c}$ whenever $A \in Q_{\alpha i}$. By [4, Lemma 5], there is a family, $\left\{T_{\alpha i A}: \alpha<\mathfrak{c}, i<2, A \in Q_{\alpha i}\right\}$, composed of pairwise disjoint sets of cardinality $\mathfrak{c}$, such that each $T_{\alpha i A}$ is a subset of $A$. For each $\alpha, i$, and $A$, let $g_{\alpha i A}: T_{\alpha i A} \rightarrow(0, \infty)$ be an arbitrary surjection. Define the function $g$ by

$$
g(x)= \begin{cases}(-1)^{i}\left(f_{\alpha} / g_{\alpha i A}\right)(x) & \text { if } x \in T_{\alpha i A}, \alpha<\mathfrak{c}, i<2, A \in Q_{\alpha i}, \\ 1 & \text { otherwise. }\end{cases}
$$

Evidently $g$ is positive. We will show that each function $f_{\alpha} / g$ is Darboux.

Let $\alpha<\mathfrak{c}, a<b$, and assume that $\left(f_{\alpha} / g\right)(a)<\left(f_{\alpha} / g\right)(b)$. (The other case is analogous.) Fix a $y \in\left(\left(f_{\alpha} / g\right)(a),\left(f_{\alpha} / g(b)\right)\right)$. We consider three cases.

If $y=0$, then $f_{\alpha}(a)<0<f_{\alpha}(b)$. So by D1, $\left(f_{\alpha} / g\right)(x)=f_{\alpha}(x)=0$ for some $x \in(a, b)$.

If $y>0$, then $f_{\alpha}(b)>0$, so $A=[a, b] \cap\left[f_{\alpha}>0\right] \neq \emptyset$. Thus $A \in Q_{\alpha 0}$. Consequently, there is an $x \in T_{\alpha 0 A} \subset[a, b]$ such that $\left(f_{\alpha} / g\right)(x)=g_{\alpha 0 A}(x)=y$.

We proceed similarly if $y<0$.

To prove the next theorem we need the following definition.

$$
\mathrm{a}(\mathcal{D}) \stackrel{\mathrm{df}}{=} \min \left\{\operatorname{card} \mathcal{F}: \mathcal{F} \subset \mathbb{R}^{\mathbb{R}} \& \neg\left(\exists_{g} \forall_{f \in \mathcal{F}} f+g \in \mathcal{D}\right)\right\} .
$$


This cardinal was defined by Natkaniec [5] and was thoroughly examined by Ciesielski and Miller [1]. It is well-known that $\mathfrak{c}<\mathrm{a}(\mathcal{D}) \leq 2^{\mathfrak{c}}[2]$. Ciesielski and Miller generalized this result by showing that $\operatorname{cf}(\mathrm{a}(\mathcal{D}))>\mathfrak{c}$. They also proved that it is pretty much all that can be said about a $(D)$ in $\mathrm{ZFC}$, by showing that $\mathrm{a}(\mathcal{D})$ can be equal to any regular cardinal between $\mathfrak{c}^{+}$and $2^{\mathfrak{c}}$, and that it can be equal to $2^{\mathfrak{c}}$ independently of the cofinality of $2^{\mathfrak{c}}$ [1]. (Actually, Ciesielski and Miller showed these results for the family of functions almost continuous in the sense of Stallings [8].)

Theorem 2.3. $\mathrm{a}(\mathcal{D})=\min \left\{\operatorname{card} \mathcal{F}: \mathcal{F} \subset(0, \infty)^{\mathbb{R}} \& \neg\left(\exists_{g} \forall_{f \in \mathcal{F}} f / g \in \mathcal{D}\right)\right\}$.

Proof. First we will prove that a $(\mathcal{D})$ is not smaller than the right-hand side of the above equality. Pick a family $\mathcal{F} \subset \mathbb{R}^{\mathbb{R}}$ such that card $\mathcal{F}=\mathrm{a}(\mathcal{D})$ and

$$
\forall_{g \in \mathbb{R}^{\mathbb{R}}} \exists_{f \in \mathcal{F}} f+g \notin \mathcal{D} \text {. }
$$

Let $\mathcal{F}^{*}=\{\exp \circ f: f \in \mathcal{F}\}$. Then $\mathcal{F}^{*} \subset(0, \infty)^{\mathbb{R}}$ and $\operatorname{card} \mathcal{F}^{*}=\operatorname{card} \mathcal{F}$. We will show that for each $g: \mathbb{R} \rightarrow \mathbb{R} \backslash\{0\}$ there is an $f^{*} \in \mathcal{F}^{*}$ such that $f^{*} / g \notin \mathcal{D}$. Let $g: \mathbb{R} \rightarrow \mathbb{R} \backslash\{0\}$. By $(1), f-\ln \circ|g| \notin \mathcal{D}$ for some $f \in \mathcal{F}$. Hence

$$
\exp \circ(f-\ln \circ|g|)=(\exp \circ f) /|g| \notin \mathcal{D},
$$

and consequently, $(\exp \circ f) / g \notin \mathcal{D}$.

The proof of the opposite inequality is analogous.

By Theorem 2.1, we have $(0, \infty)^{\mathbb{R}} \subset \mathcal{D} / \mathcal{D}$. So, we obtain the following corollary.

Corollary 2.4. $\mathrm{q}(\mathcal{D}) \leq \mathrm{a}(\mathcal{D})$.

For a partially ordered set $(\mathbb{P}, \leq)$, we say that $G \subset \mathbb{P}$ is a $\mathbb{P}$-filter, if

- for all $p, q \in G$, there exists $r \in G$ with $r \leq p$ and $r \leq q$, and

- for all $p, q \in \mathbb{P}$, if $p \in G$ and $p \leq q$, then $q \in G$.

Define $D \subset \mathbb{P}$ to be dense, if for every $p \in \mathbb{P}$ there exists $q \in D$ with $q \leq p$.

For a cardinal $\kappa$ and a poset $\mathbb{P}$, define the following statements (Martin's Axiom for $\mathbb{P}$ and Lusin's Axiom for $\mathbb{P})$ :

$\mathrm{MA}_{\kappa}(\mathbb{P})$ : for any family $\mathfrak{D}$ of dense subsets of $\mathbb{P}$ with card $\mathfrak{D}<\kappa$, there exists a $\mathbb{P}$-filter $G$ such that $D \cap G \neq \emptyset$ for every $D \in \mathfrak{D}$. 
$\operatorname{Lus}_{\kappa}(\mathbb{P})$ : there exists a sequence $\left\langle G_{\alpha}: \alpha<\kappa\right\rangle$ of $\mathbb{P}$-filters, called a $\kappa$-Lusin sequence, such that for every dense set $D \subset \mathbb{P}$

$$
\operatorname{card}\left\{\alpha<\kappa: G_{\alpha} \cap D=\emptyset\right\}<\kappa .
$$

From now on, let

$$
\mathbb{P}=\left\{p \in(0, \infty)^{X}: X \subset \mathbb{R} \& \operatorname{card} X<\mathfrak{c}\right\} .
$$

Define $p \leq q$ if $q \subset p$, i.e., if $p$ extends $q$ as a partial function.

The proof of the next theorem is actually a repetition of argument used by Ciesielski and Miller [1, Lemma 3.1].

Theorem 2.5. $\mathrm{MA}_{\kappa}(\mathbb{P})$ implies $\mathrm{q}(\mathcal{D}) \geq \kappa$.

Proof. Assume $\mathrm{MA}_{\kappa}(\mathbb{P})$. By Theorem 2.2, we may assume that $\kappa>\mathfrak{c}$.

First observe that for every $x \in \mathbb{R}$, the set $D_{x}=\{p \in \mathbb{P}: x \in \operatorname{dom} p\}$ is dense in $\mathbb{P}$. Indeed, let $x \in \mathbb{R}$ and $p \in \mathbb{P}$. If $x \in \operatorname{dom} p$, then put $q=p$; otherwise let $q=p \cup\{(x, 1)\}$. Clearly $q \in D_{x}$ and $q \leq p$.

Now we will show that for any $f \in \mathcal{D} / \mathcal{D}, y \neq 0$, and $a<b$, if the set $[a, b] \cap[f / y>0]$ is nonempty, then the set

$$
D_{\text {fyab }}=\left\{p \in \mathbb{P}: \exists_{x \in[a, b] \cap \operatorname{dom} p} p(x)=f(x) / y\right\}
$$

is dense in $\mathbb{P}$. Let $p \in \mathbb{P}, f \in \mathcal{D} / \mathcal{D}, y>0, a<b$, and assume that $[a, b] \cap$ $[f / y>0] \neq \emptyset$. Since $f$ satisfies D2, $\operatorname{card}([a, b] \cap[f / y>0])=\mathfrak{c}$. We have card $\operatorname{dom} p<\mathfrak{c}$; so there is an $x \in(a, b) \cap[f / y>0] \backslash \operatorname{dom} p$. Then the function $q=p \cup\{(x, f(x) / y)\}$ satisfies $q \in D_{\text {fyab }}$ and $q \leq p$.

To show that $\mathrm{q}(\mathcal{D}) \geq \kappa$ pick a family of functions $\mathcal{F} \subset \mathcal{D} / \mathcal{D}$ with card $\mathcal{F}<\kappa$. Define

$$
\mathfrak{D}=\left\{D_{x}: x \in \mathbb{R}\right\} \cup\left\{D_{\text {fyab }}: f \in \mathcal{F}, y \neq 0, a<b,[a, b] \cap[f / y>0] \neq \emptyset\right\} .
$$

Then $\mathfrak{D}$ is a family of dense subsets of $\mathbb{P}$ and $\operatorname{card} \mathfrak{D}<\kappa$. Applying $\mathrm{MA}_{\kappa}(\mathbb{P})$ we can find a $\mathbb{P}$-filter $G$ which meets every $D \in \mathfrak{D}$.

Let $g=\bigcup G$. Evidently $g$ is a function and $g$ is positive. For every $x \in \mathbb{R}$, we have $D_{x} \cap G \neq \emptyset$; so $\operatorname{dom} g=\mathbb{R}$. We will show that each $f / g$ is Darboux. Let $f \in \mathcal{F}, a<b$, and assume that $(f / g)(a)<(f / g)(b)$. (The other case is analogous.) Fix a $y \in((f / g)(a),(f / g)(b))$. We consider three cases. If $y=0$, then $f(a)<0<f(b)$. So by D1, $(f / g)(x)=f(x)=0$ for some $x \in(a, b)$. If $y>0$, then $f(b)>0$, so $[a, b] \cap[f / y>0] \neq \emptyset$. Since $D_{\text {fyab }} \cap G \neq \emptyset$, there are a $p \in G$ and an $x \in[a, b] \cap \operatorname{dom} p$ such that $p(x)=f(x) / y$. Then $(f / g)(x)=(f / p)(x)=y$.

We proceed similarly if $y<0$. 
To prove the next theorem we will use two other posets. Let

$$
\mathbb{P}^{\prime}=\left\{p \in \mathbb{R}^{X}: X \subset \mathbb{R} \& \operatorname{card} X<\mathfrak{c}\right\},
$$

and $p \leq q$ iff $q \subset p$. Moreover let

$$
\mathbb{P}^{*}=\left\{(p, \mathcal{E}): p \in \mathbb{P}^{\prime}, \mathcal{E} \subset \mathbb{R}^{\mathbb{R}} \& \operatorname{card} \mathcal{E}<\mathfrak{c}\right\},
$$

and define $(p, \mathcal{E}) \leq(q, \mathcal{F})$ iff

$$
q \subset p, \mathcal{E} \supset \mathcal{F} \text {, and } p(x) \neq f(x) \text { for all } x \in \operatorname{dom} p \backslash \operatorname{dom} q \text { and } f \in \mathcal{F} \text {. }
$$

Theorem 2.6. Suppose that $\kappa>\mathfrak{c}, \kappa$ is regular, and $\operatorname{Lus}_{\kappa}\left(\mathbb{P}^{*}\right)$ holds. Then $\mathrm{q}(\mathcal{D})=\mathrm{a}(\mathcal{D})=\kappa$.

Proof. The inequality $\mathrm{q}(\mathcal{D}) \leq \mathrm{a}(\mathcal{D})$ follows by Corollary 2.4. The inequality $\mathrm{a}(\mathcal{D}) \leq \kappa$ follows by [1, Lemma 3.2 and Theorem 2.1]. By [1, Lemma 3.3], $\operatorname{Lus}_{\kappa}\left(\mathbb{P}^{*}\right)$ implies $\mathrm{MA}_{\kappa}\left(\mathbb{P}^{\prime}\right)$. But the posets $\mathbb{P}$ and $\mathbb{P}^{\prime}$ are order isomorphic; so $\mathrm{MA}_{\kappa}(\mathbb{P})$ holds. Now the inequality $\mathrm{q}(\mathcal{D}) \geq \kappa$ follows by Theorem 2.5.

Ciesielski and Miller proved that the assumptions of Theorem 2.6 are independent of ZFC [1]. So, we have the following problem.

Problem. Can the equality $\mathrm{q}(\mathcal{D})=\mathrm{a}(\mathcal{D})$ be proved in ZFC?

\section{References}

[1] K. Ciesielski and A. W. Miller, Cardinal invariants concerning functions whose sum is almost continuous, Real Anal. Exchange, 20 (1994-95), no. 2, 657-672.

[2] H. Fast, Une remarque sur la propriété de Weierstrass, Colloq. Math., 7 (1959), 75-77.

[3] J. Jałocha, Quotients of quasi-continuous functions, J. App. Anal., 6 (2000), no. 2, to appear.

[4] A. Maliszewski, Sums of bounded Darboux functions, Real Anal. Exchange, 20 (1994-95), no. 2, 673-680.

[5] T. Natkaniec, Almost continuity, Real Anal. Exchange, 17 (1991-92), no. 2, 462-520.

[6] T. Natkaniec, Products of Darboux functions, Real Anal. Exchange, 18 (1992-93), no. 1, 232-236. 
[7] T. Natkaniec and W. Orwat, Variations on products and quotients of Darboux functions, Real Anal. Exchange, 15 (1989-90), no. 1, 193-202.

[8] J. Stallings, Fixed point theorem for connectivity maps, Fund. Math., 47 (1959), 249-263. 